\title{
Ecological landscapes guide the assembly of optimal microbial communities
}

\author{
Ashish B. George ${ }^{1,2,3, *}$ and Kirill S. Korolev ${ }^{1,2,4, *}$ \\ 1 Department of Physics, Boston University, Boston, MA 02215, USA \\ 2 Biological Design Center, Boston University, Boston, MA 02215, USA \\ 3 Carl R. Woese Institute for Genomic Biology and Department of Plant Biology, \\ University of Illinois at Urbana-Champaign, Urbana, IL 61801, USA \\ ${ }^{4}$ Graduate Program in Bioinformatics, Boston University, Boston, MA 02215, USA \\ * Correspondence to: ashish.b.george@gmail.com, korolev@bu.edu
}

December 14, 2021

\begin{abstract}
Assembling optimal microbial communities is key for various applications in biofuel production, agriculture, and human health. Finding the optimal community is challenging because the number of possible communities grows exponentially with the number of species, and so an exhaustive search cannot be performed even for a dozen species. A heuristic search that improves community function by adding or removing one species at a time is more practical, but it is unknown whether this strategy can discover an optimal or nearly optimal community. Using consumer-resource models with and without cross-feeding, we investigate how the efficacy of search depends on the distribution of resources, niche overlap, cross-feeding, and other aspects of community ecology. We show that search efficacy is determined by the ruggedness of the appropriately-defined ecological landscape. We identify specific ruggedness measures that are both predictive of search performance and robust to noise and low sampling density. The feasibility of our approach is demonstrated using experimental data from a soil microbial community. Overall, our results establish the conditions necessary for the success of the heuristic search and provide concrete design principles for building high-performing microbial consortia.
\end{abstract}

Keywords: community assembly, microbiome, consumer-resource model, community design, crossfeeding

\section{Introduction}

Life on earth is sustained by a myriad of biochemical transformations. From synthesis to decomposition, microbial communities perform the bulk of these transformations, including photosynthesis, nitrogen fixation, and the digestion of complex molecules [1-3]. This understanding has generated considerable interest in exploiting microbial capabilities for producing energy and food, degrading waste, and improving health [4-10].

The metabolic ingenuity of microbial communities can be harnessed by simply placing the right 
combination of species in the right environment. Yet, the search for the appropriate species and environmental conditions is anything but simple. Ecological interactions are nonlinear and often unpredictable, so an exhaustive search across all relevant variables is the only sure way to build a community with the best performance. Such an exhaustive search is however infeasible because the number of possible combinations increases exponentially with the number of species and environmental factors; just 16 species leads to over 65,000 combinations. Even with expert knowledge, computer simulations, and liquid-handling robots, exhaustive testing remains infeasible for any complex microbial community [11-19].

This challenge is not unique to ecology. In fact, most optimization problems cannot be solved directly, and one has to resort to heuristic search strategies [20, 21]. For microbial communities, a simple heuristic search (a greedy gradient-ascent) proceeds via a series of steps. At each step, a set of new communities is created by adding or removing one or a few species from the current microbial community. The community with the best performance is then chosen for the next step. Although easy to implement, the search can get stuck at a local optimum and achieve only a small fraction of the best possible performance. It is therefore essential to identify when such heuristic search is likely to succeed.

Here, we study the success of the heuristic search by simulating a range of realistic and widelyused consumer-resource models of microbial communities, which have reproduced many features of natural and experimental communities [22-24]. In the simulated communities, microbes compete for externally supplied resources and potentially produce other metabolites that can be exchanged among the community members. Our simulations allowed us to vary the complexity of the microbial ecosystem and explore its effects on the efficacy of the search. We identified specific ecological properties, such as the average niche overlap, to be highly predictive of the search success.

To integrate our findings into a coherent framework and understand when search fails, we define and analyze community function landscapes utilizing an analogy to the fitness landscapes from population genetics [25]. The community function of interest is analogous to fitness, and the composition of the community is analogous to the genome. The heuristic search then corresponds to an uphill walk on this multi-dimensional landscape. Similar to previous studies in evolution, we found that search success depends on the ruggedness of the ecological landscape. Multiple definitions of ruggedness exist, and we identified those that are more predictive across diverse ecological scenarios and can be reliably estimated from limited, noisy experimental data. The intuition gained from our numerical studies applied well to real experimental data on an ecological landscape for six soil microbes from Ref. [26].

Overall, our work identifies the connections between ecological properties, community structure, and optimization. This knowledge makes it possible to predict whether heuristic search is feasible and provides concrete strategies to increase the chance of success by adjusting the environment or the pool of candidate species. Furthermore, we anticipate that community landscapes can provide a unifying framework to compare microbial consortia and their assembly across diverse ecological settings. 


\begin{tabular}{|c|c|}
\hline Outcome of dynamics & Models and experiments \\
\hline Single steady state & $\begin{array}{l}\text { Consumer-resource models with substitutable resources: }[27-31] \text {. } \\
\text { Consumer-resource models with cross-feeding: }[32,33] \text {. } \\
\text { Many Lotka-Volterra models including those with random interactions } \\
\text { with low to moderate variance: }[34-39] \\
\text { Compatible empirical observations: }[40-42] .\end{array}$ \\
\hline $\begin{array}{l}\text { Typically a single steady } \\
\text { state }\end{array}$ & $\begin{array}{l}\text { Consumer resource models with non-substitutable resources or species } \\
\text { consuming resources diauxically: }[43,44] . \\
\text { Compatible empirical observations: }[45,46] \text {. }\end{array}$ \\
\hline Multiple steady states & $\begin{array}{l}\text { Lotka-Volterra models with random interactions with large variance: }[34 \text {, } \\
35] \text {. } \\
\text { Compatible empirical observations: }[47,48] \text {. }\end{array}$ \\
\hline $\begin{array}{l}\text { Complex dynamics and } \\
\text { chaos }\end{array}$ & $\begin{array}{l}\text { Stochastic neutral models: }[49,50] \text {. } \\
\text { Consumer resource models with highly nonlinear uptake of non- } \\
\text { substitutable resources: }[28,31,51] \text {. } \\
\text { Lotka-Volterra models with antisymmetric interactions or random inter- } \\
\text { actions with large variance: }[34,52] \text {. } \\
\text { Compatible empirical observations: }[53] .\end{array}$ \\
\hline
\end{tabular}

Table 1: Microbial communities often converge to a unique steady state, but more complex dynamics are possible. Macroscopic organisms are discussed in Ref. [54]. 


\section{Model}

We simulated microbial communities using consumer-resource models with and without crossfeeding $[27,32]$. Compared to the commonly-used Lotka-Volterra models, our approach is more realistic and makes a closer connection to the metabolic interactions that underpin many optimization problems in biotechnology [55-59]. Furthermore, it is much easier to make educated guesses about the production and consumption of resources than interaction coefficients. One disadvantage of our approach is that other interactions, such as due to various antimicrobials, are excluded. Although such interactions play a prominent role in natural communities, they are often removed or otherwise controlled in engineered communities.

We assume that the growth of each microbe depends on its ability to consume various available resources and convert consumed resources into biomass. The resources could be externally supplied or excreted by the microbes themselves as metabolic byproducts [32,60-63]. Both the species and the resources are diluted at a fixed rate to prevent the accumulation of nutrients and biomass, as in a chemostat. The mathematical details of the models are further explained in Methods. Numerical simulations were carried out using code based on the 'Community Simulator' package [64].

The ecological dynamics could, in principle, be time-dependent or sensitive to the species abundances at the beginning of the experiment. For consumer-resource models, however, the community always reaches a unique steady-state that depends only on the initial presence or absence of species. Recent studies show that time-dependence and multi-stability are more of an exception than the norm and most (especially engineered) communities exhibit the simple behavior of consumer-resource models (see Table 1 and Ref. [65-67]).

The search for the optimal community begins with a choice of the desired ecological function, denoted as $\mathcal{F}$; this could be the production rate of a particular metabolite, the degradation rate of an undesirable compound, or even the diversity of the community. One also needs to choose the pool of $S$ candidate species from which to assemble the microbial community. In total, there are $2^{S}$ possible communities, corresponding to distinct species combinations that can be labeled by $\vec{\sigma}$, a string of ones and zeros denoting the presence or absence of each species in the starting community (Fig. 1). The simplest search protocol starts with one of the $2^{S}$ possibilities, for e.g., the community with all species present. Then, every step of the search is a heuristic gradient-ascent, which considers the current community and the $S$ neighboring communities obtained by changing each of the elements of $\vec{\sigma}$, i.e., adding or removing a species. These communities are simulated until they reach a steady state, and the community with the highest value of $\mathcal{F}$ is taken to be the next current state. The process repeats until the community function cannot be improved any further by adding or removing a single species. We also explore several variations of this search protocol after obtaining the main results in the context of the search protocol just described.

Because consumer-resource models reach a unique steady state, all quantities that depend on species and resource concentrations are uniquely determined by the presence-absence of species at the beginning of the simulation. Thus, the community function is fully determined by $\vec{\sigma}$. The search for the optimal community then reduces to the maximization of $\mathcal{F}$ over $\vec{\sigma}$, and $\mathcal{F}(\vec{\sigma})$ can be viewed as a multi-dimensional ecological or community function landscape. Mathematically, this landscape is related to the fitness landscapes studied in population genetics [25], with $\mathcal{F}$ analogous to fitness and $\vec{\sigma}$ analogous to genotype. Below, we explore the structure of ecological landscapes under different ecological conditions and examine the influence of landscape structure on the success of 


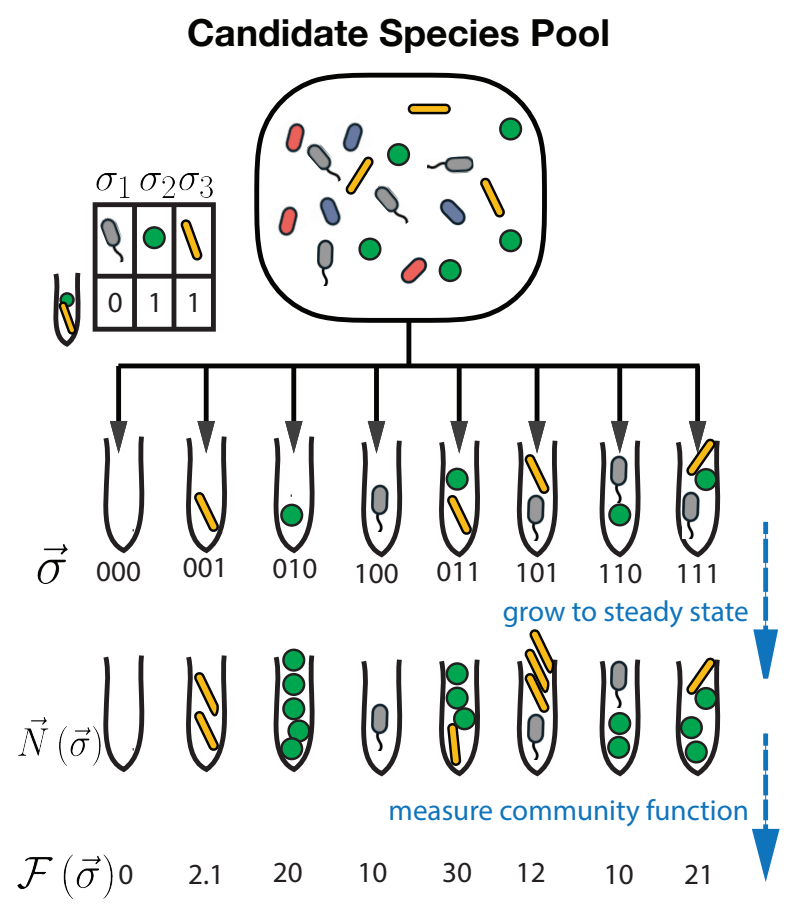

Figure 1: An ecological landscape framework for community optimization. We consider the problem of assembling an ecological community from a pool of $S$ candidate species that is optimal at performing a desired function. The candidate species in the pool are indexed from 1 to $S$. A test community is seeded with a subset of the candidate species. The composition of the community is encoded in $\vec{\sigma}$ using 0 for absence and 1 for presence for each of the species. The test communities are allowed to grow until they reach a steady state. The species abundances in the steady-state community is $\vec{N}(\vec{\sigma})$, and the community function is $\mathcal{F}(\vec{\sigma})$.

the heuristic search.

\section{Results}

\section{Niche overlap controls the complexity of ecological landscapes}

The problem of finding optimal microbial communities brings a number of questions about the landscape structure and its influence on search success. Before we begin to explore these questions in detail, it is convenient to establish how one can control the difficulty of the search by varying a relevant ecological variable. Intuition suggests that communities with many inter-specific interactions should be more complex than communities where species are largely independent from each other. Therefore, we explored how the density of metabolic interactions influences community structure.

The density of interactions is controlled by the overlap in the resource utilization profiles of the species in the community. Simply put, the more species consume any given resource, the greater the number of interactions. In our model, these interactions are encoded in the consumption matrix; see Fig. 2A. We created these matrices by allowing each species to consume only a random subset 
of $M_{\text {consumed }}$ resources out of $M_{\text {total }}$ resources supplied externally.

When $M_{\text {consumed }} \approx M_{\text {total }}$, the species are generalists; several species are consuming each of the resources, and the niche overlap is high (Fig. 2B). In the opposite limit $\left(M_{\text {consumed }} \ll M_{\text {total }}\right)$, species are specialists; most species are consuming different resources, and the niche overlap is low. Thus, $M_{\text {consumed }} / M_{\text {total }}$ controls the density of the interactions and could influence the ruggedness of the community landscape.

To gain a broader view of the community structure, we opted not to consider a specific ecological function, but instead looked at the abundances of all species in the community. Thus, we studied the assembly map, $\vec{N}(\vec{\sigma})$, from initial composition to steady-state abundances. Since optimization is easy for linear problems, we quantified the linearity of $\vec{N}(\vec{\sigma})$ by fitting the following model to the data

$$
N_{i}(\vec{\sigma})= \begin{cases}\sum_{j=1}^{S} A_{i j} \sigma_{j}+\epsilon_{i}, & \text { if } \sigma_{i}=1 \\ 0 & \text { if } \sigma_{i}=0\end{cases}
$$

where $\epsilon_{i}$ is the model error, and $A_{i j}$ are determined via the least-squares regression.

The differences between the actual and predicted values of the abundance of species $i$ across all possible communities can be used to compute the coefficient of determination $R_{i}^{2}$. The performance of the linear model in predicting the abundance of all species in the community is then characterized by the average of $R_{i}^{2}$ across all the species. This average $\overline{R^{2}}$ is close to zero when the linear model fails to fit the data; $\overline{R^{2}}$ is close to one when the assembly map is approximately linear and species abundances are explained well by independent contributions from other community members.

As expected, Fig. 2 shows that community assembly becomes simpler as the number of interspecific interactions decreases. For low niche overlap, we obtained good linear fits and large values of $\overline{R^{2}}$. In contrast, $\overline{R^{2}}$ was small when niche overlap was high. This behavior was consistent across different numbers of candidate species and resources, so we chose the niche overlap as the default method to control community structure. We also checked whether our conclusions are robust by changing environmental complexity or by including metabolic cross-feeding; see the results below and SI Fig. S1.

\section{The role of community function}

The optimization process could be affected not only by the interspecific interactions, but also by the community function that is being maximized. To investigate this possibility, we studied the efficacy of the search process for three choices of the community function described below. Since consumer-resource models simplify details of microbial metabolism, we did not explicitly model the production of a desired metabolite. Instead, we followed experimental studies in expressing the desired community function in terms of species abundances in the community $[68,69]$.

The three community functions were chosen to cover scenarios ranging from functions dependent on the contributions of many species to functions dependent on just a single species. The first community function (diversity) was the diversity of the community. We explored this function 

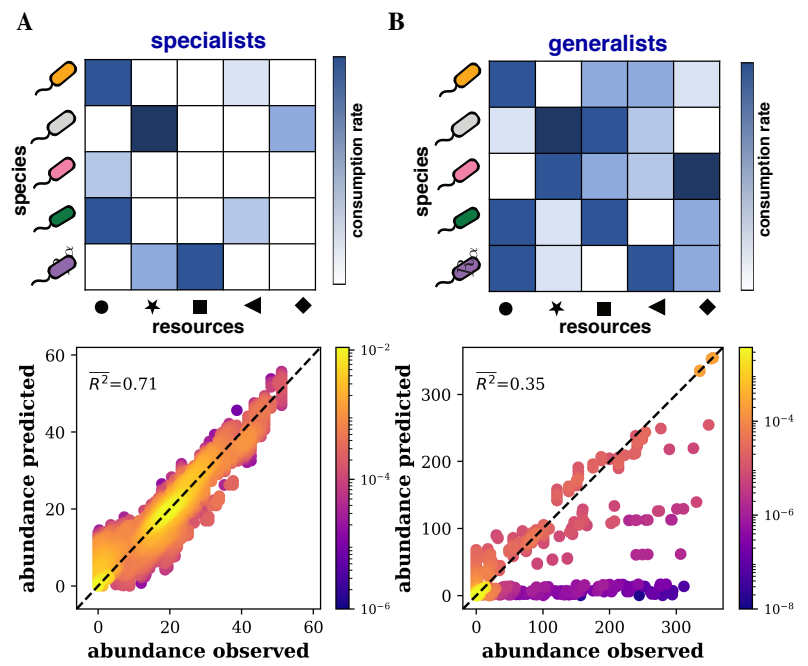

C

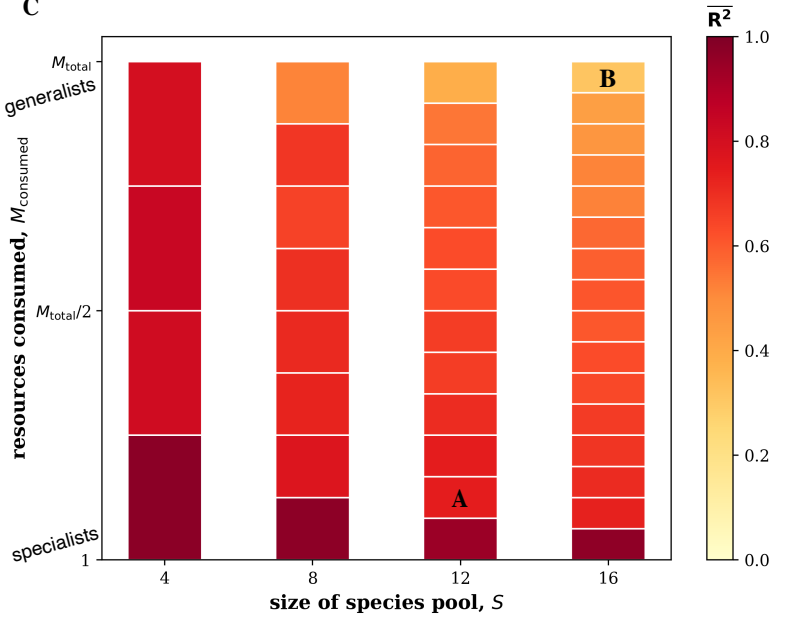

Figure 2: Niche overlap determines the linearity of ecological landscapes. The top panels in (A) and (B) show the consumption matrices for species in each species pool. In (A), the niche overlap is low because the species are specialists and consume only a few resources. In (B), the niche overlap is high because the species are generalists and consume many resources. The bottom panels show how well a linear model (Eq. 5) fits the steady-state abundances across all possible species combinations. The goodness of the model fit, quantified by $\overline{R^{2}}$, is higher when the niche overlap is lower. This conclusion is robust to varying the size of the community and the degree of niche overlap. (C) shows how $\overline{R^{2}}$ varies with the number of species in the pool, $S$, and the average

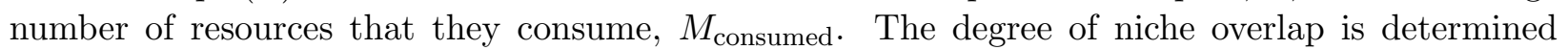
by $M_{\text {consumed }} / M_{\text {total }}$. Letters indicate the parameters used in panels $\mathrm{A}$ and $\mathrm{B}$. Note that $\overline{R^{2}}$ was computed by averaging $R^{2}$ of the linear model fitted separately for each species, and the number of resources supplied was fixed to $M_{\text {total }}=S$. For each set of parameters, results were averaged over 10 independently generated species pools. Other simulation parameters are provided in Methods, and the robustness of the conclusions to certain modeling assumptions is further discussed in the SI. 

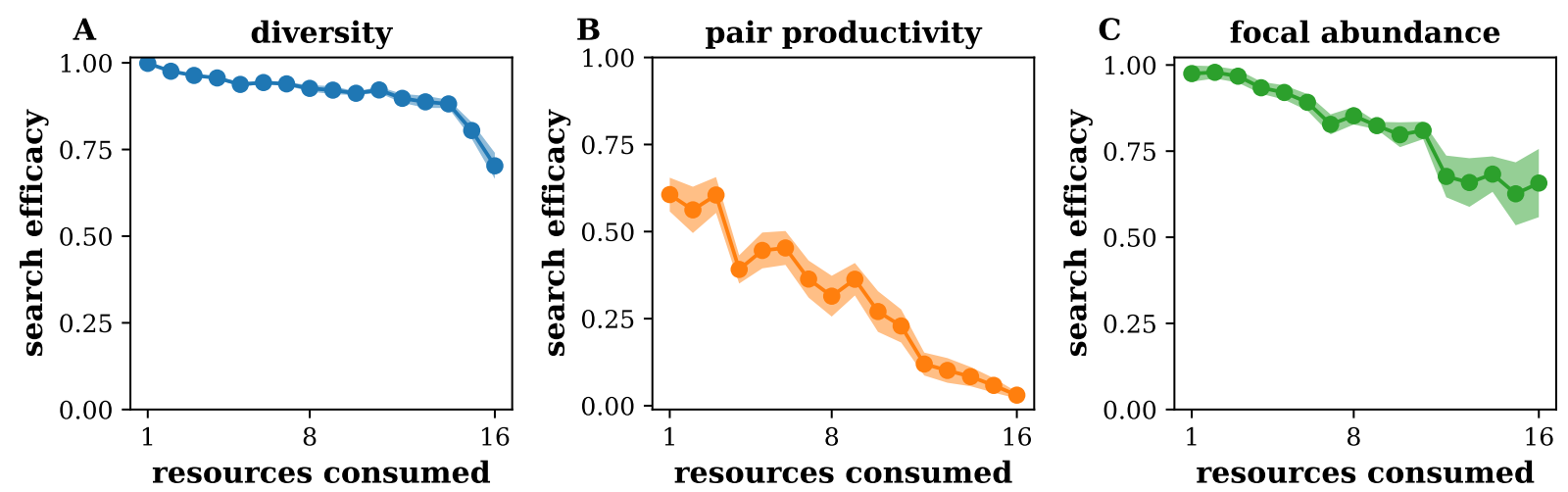

Figure 3: Search efficacy decreases with niche overlap. Search efficacy was quantified as the ratio of the best community function found by the search $\mathcal{F}_{\text {found }}$ to the highest value $\mathcal{F}_{\text {max }}$ across the entire landscape. The plots report the average and the standard error of the mean obtained from ten independently generated species pools. Different panels correspond to different community functions. The complexity of the landscape was controlled by varying the average number of resources that each species consumed $M_{\text {consumed }}$ while keeping the total number of resources $M_{\text {total }}$ fixed at $M_{\text {total }}=S=16$. See Methods for a complete description of these simulations.

because it could be predictive of the overall community productivity in some ecosystems [70-73]. The precise definition of the diversity did not affect our conclusions, so we focused on the Shannon Diversity (Eq. 7). The second community function (pair productivity) was the product of the abundances of two specific microbes (Eq. 8). This choice was motivated by the communities where the combined efforts of two species ware necessary to produce a metabolite. The third community function (focal abundance) was simply the abundance of a given species, which could reflect the production or degradation of a specific metabolite.

We quantified the search efficacy as the ratio of the best community function found by the search $\mathcal{F}_{\text {found }}$ to the highest value $\mathcal{F}_{\max }$ across the entire landscape. To reduce the influence of the starting community, we report the search efficacy averaged over all possible starting communities.

Qualitatively, the results were the same for all three community functions: The efficacy of search decreased with increasing niche overlap (Fig. 3). We however found important quantitative differences. While optimization of diversity showed only a modest drop with higher niche overlap, the search efficacy for pair productivity plummeted to near zero. This sharp drop was due to sporadic coexistence of the two species. When the two species do not coexist in a region of the landscape, the community function is zero in that neighborhood and the search cannot progress. This challenge can be partly overcome by starting search from the best out of many random communities instead of a single one, which we discuss in Fig. 9.

\section{Ruggedness of ecological landscapes}

Niche overlap is only one of many mechanisms that can produce ecological landscapes of varying complexity. For example, cross-feeding could lead to additional interactions not captured by the consumption matrix. It would therefore be quite valuable to develop a mechanism-free metric of landscape complexity. In population genetics and optimization theory, such metrics are known as 
landscape ruggedness [74-76]. Rugged landscapes with many scattered peaks are much harder to navigate than smooth landscapes with a single peak. Many measures of ruggedness exist, and some of the most commonly used ones are described in the SI. In the main text, we limit the discussion to the three metrics that we found to be the most useful for microbial communities. These metrics are described below, and their mathematical definitions are provided in Methods.

The roughness-slope ratio $r / s$ quantifies landscape linearity [76, 77]. The roughness, $r$, measures the error of a linear fit to the community function landscape and the slope, $s$, measures the average magnitude of the change in $\mathcal{F}$ when a species is added or removed. Small values of $r / s$ indicate that there is a strong trend that is slightly obscured by local fluctuations. Such landscape are easy to navigate because the heuristic search can detect the direction of the gradient. The exact opposite occurs for large $r / s$ because large point-to-point fluctuations obscure the path towards the optimum.

A related, but different approach is to capture the changes in the community function upon adding or removing a single species. This metric is defined as $1-Z_{\mathrm{nn}}$, where $Z_{\mathrm{nn}}$ is the average correlation between the values of $\mathcal{F}$ in communities that differ by adding or removing a single species. Large values of $1-Z_{\mathrm{nn}}$ indicate that changes in community function are uncorrelated and the search is difficult.

The third metric, $F_{\text {neut }}$, captures the challenge of quasi-neutral directions. If changes in $\sigma$ leave the community function nearly the same, then it is difficult to determine how to modify the community to increase $\mathcal{F}$. This situation occurs when the added species fails to establish or fail to affect the function of interest despite invading. Quasi-neutral directions are common in some landscapes and are known to be a major impediment to optimization [78-80]. Thus, the fraction of quasi-neutral directions $F_{\text {neut }}$ could be a valuable predictor of the search success.

We analyzed how these three metrics change with the niche overlap across different community functions. In all cases, we found that landscape ruggedness increases with the number of interspecific interactions. The plots in Fig. 4 are representative of the behavior that we observed. Note that the magnitude and pace of increase varied among the metrics suggesting that there could be important differences in their ability to predict the efficacy of the heuristic search.

We also examined potential limitations of ruggedness metrics. Because they map the entire ecological landscape into a single number, ruggedness measures may not fully capture the actual structure of the search space. Hence, we looked for a ruggedness metric that captures the landscape with more than just a single number. Moreover, we sought to capture nonlinear effects, which are typically ignored by the commonly-used measures of ruggedness. To capture higher-order effects, we examined the fits of $\mathcal{F}(\vec{\sigma})$ by models of increasing complexity. Starting with the linear model, we added quadratic, cubic, quartic, etc terms in $\vec{\sigma}$ (see Methods). These terms account for nonpairwise interactions, e.g. interactions influenced by other species. Such conditional interactions could occur via a variety of mechanisms; for example, a species can induce an interaction between two other species by producing a nutrient that is consumed by both of these other species.

The model errors (unexplained variance) decreased approximately exponentially with the model complexity (Fig. 5). The rate of decrease was similar for smooth and rugged landscapes with rugged landscapes always requiring a more complex model to achieve similar accuracy. We found no compelling examples to justify characterizing landscape ruggedness by more than a single number. 

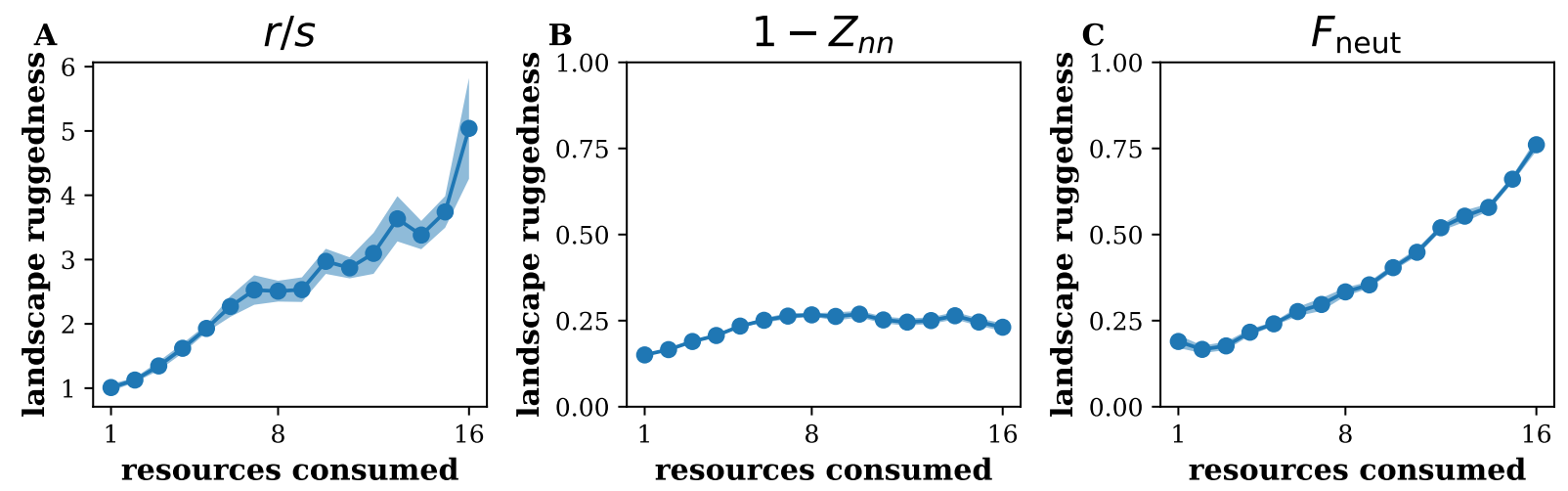

Figure 4: Ruggedness of community function landscapes. All metrics of ruggedness increased with niche overlap. Different panels correspond to different metrics of ruggedness. The plots report the average and the standard error of the mean obtained from ten independently generated species pools. The complexity of the landscape was controlled by varying the average number of resources that each species consumed, $M_{\text {consumed, }}$ while keeping the total number of resources, $M_{\text {total }}$, fixed at $M_{\text {total }}=S=16$. See Methods for the complete description of these simulations. The community function here was community diversity; see SI Fig. S2 for another community function.

Therefore, we focused on the simple metrics of landscape ruggedness defined above.

\section{Search is harder on rugged landscapes}

Although optimization should be more difficult on rugged landscapes, it is still necessary to quantify this relationship and determine which, if any, of the ruggedness measures can be used in realworld applications. So, we evaluated the search efficacy for the landscapes from Fig. 4. All three ruggedness metrics were significantly anti-correlated with the search efficacy $\left(p \ll 10^{-4}\right)$.

The examples of these correlations are shown see Fig. 6, which also illustrates how the strength of the correlation varies with the metric and community function. Notably, the roughness-slope ratio consistently showed the strongest anti-correlation, and ruggedness measures were more predictive of functions determined by groups of interacting species. Overall, we found that landscape ruggedness is a good predictor of the search success across all situations examined.

\section{Landscape ruggedness can be estimated from limited data}

So far, all of our results were obtained with the full knowledge of the ecological landscape. In practical applications, however, one can examine only a very limited set of species combinations. Thus, it is important to determine whether the ruggedness of a landscape can be determined from limited data.

We estimated different ruggedness metrics for various fractions of the complete landscapes. This was done differently for different metrics because some of them require information on the nearest neighbors while others do not; see Methods. The results showed that ruggedness can indeed be well estimated from as little as $0.1 \%$ of the total number of possible communities (Fig. 7A,B). Moreover, the estimated ruggedness remained highly informative of search success (Fig. 7C). The practical 


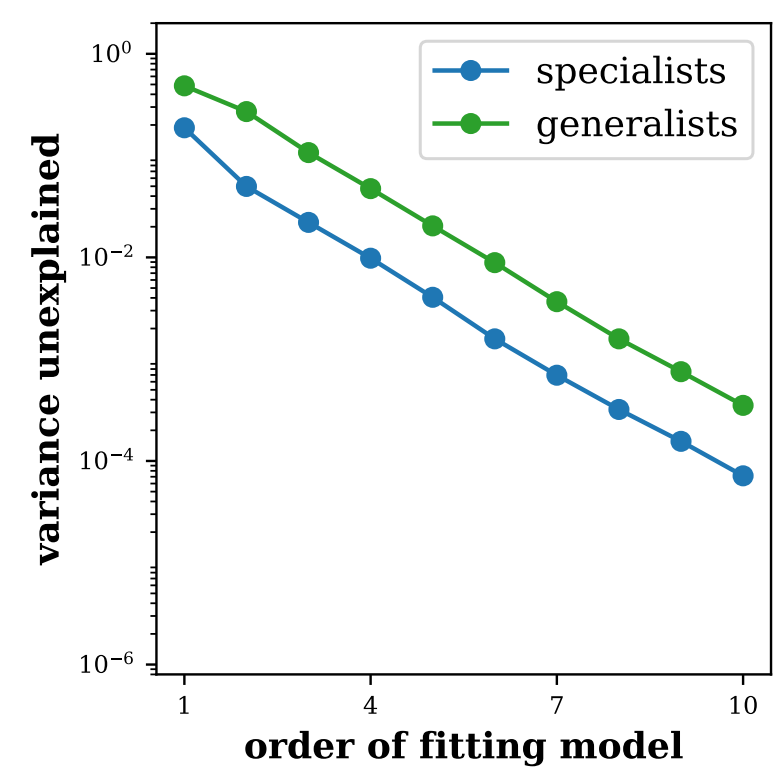

Figure 5: Contribution of higher-order interactions. Including higher order terms in the fitting model reduced the variance in the data not explained by the fit. First order fitting models are linear, second - quadratic, etc. The decrease in unexplained variance was approximately exponential. We used $S=16, M_{\text {consumed }}=2$ for specialists and $M_{\text {consumed }}=14$ for generalists; the community function was Shannon diversity. All other simulation parameters are the same as in Fig. 4.
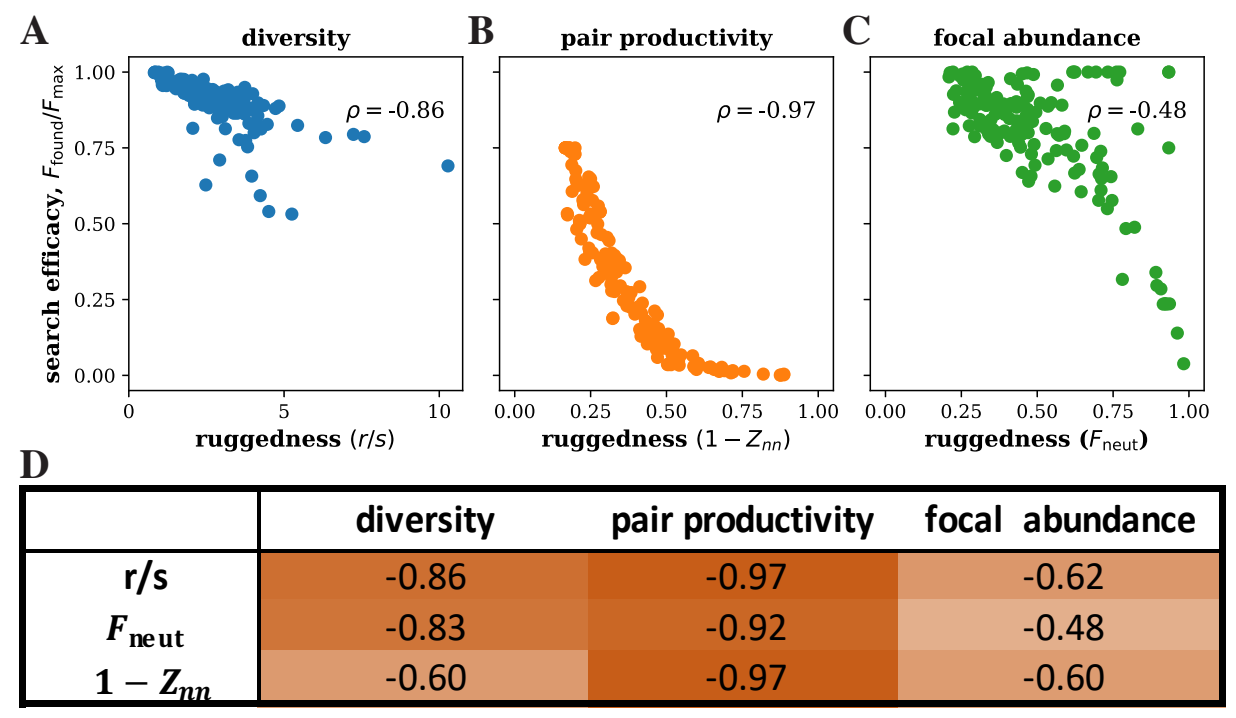

Figure 6: Landscape ruggedness inhibits search success. Search efficacy is anti-correlated with the landscape ruggedness. Panels (A), (B), (C) show examples for different community functions and ruggedness metrics. Each point corresponds to a separate $S=16$ species pool studied in Fig. 4. The systematic effects of the ruggedness metric and the type of community function is explored in (D), which reports the Spearman's correlation coefficient $\rho$ between search efficacy and ruggedness. The correlations were highly significant $\left(p \ll 10^{-4}\right)$ in all cases. 

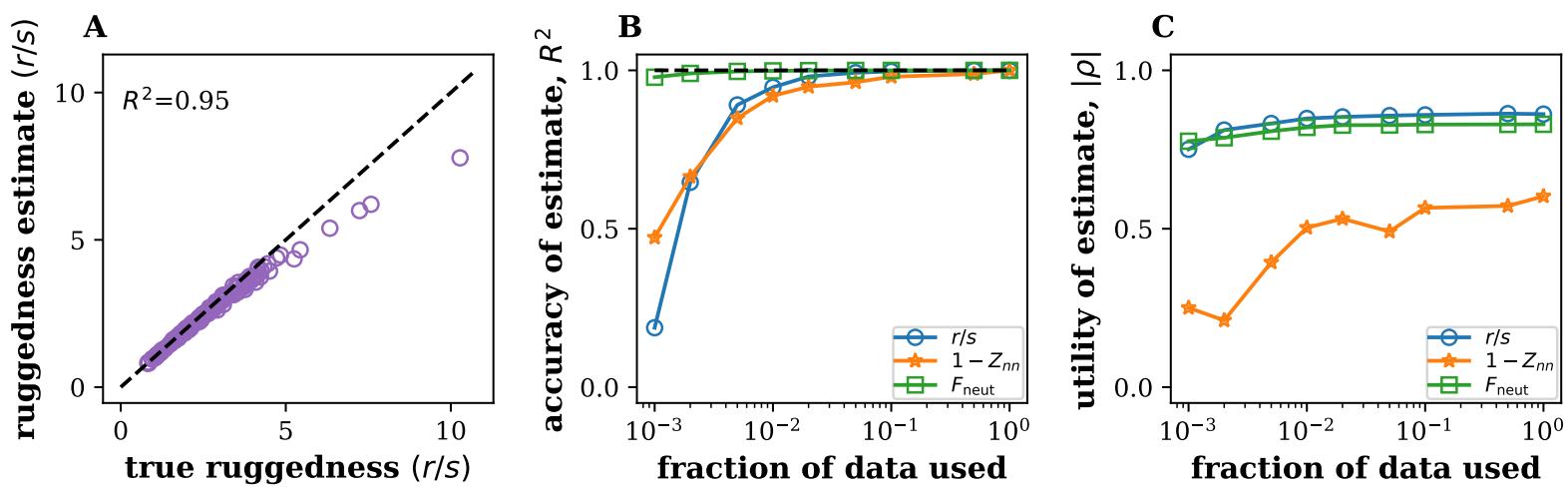

Figure 7: Estimating ruggedness from limited data. (A) True and estimated ruggedness are tightly correlated when only $1 \%$ of the data is available for the estimation. The data are from Fig. 4, and each point corresponds to a landscape with a different degree of niche overlap. (B) The accuracy of the estimation improves with the amount of available data for all ruggedness measures. (C) The estimated ruggedness remain highly informative of search efficacy. The utility of the ruggedness estimate, $|\rho|$, is measured as the magnitude of the Spearman's correlation coefficient between search efficacy and estimated ruggedness. Note that $|\rho|$ remained close to one even for very low fractions of the data for which $R^{2}$ in panel $\mathrm{B}$ started to decrease rapidly suggesting that deviations between predicted and actual roughness (see A and B) do not impede the prediction of search success. For this figure, the community function is the Shannon diversity. Similar results were obtained for other functions tested.

utility of ruggedness estimates was further confirmed by their robustness to the measurement noise (Fig. S3).

\section{Results generalize to other models and search protocols}

We further tested the utility of ruggedness measures by applying them to different models and search protocols.

The standard consumer-resource models are undeniably simpler than real microbial communities and have certain mathematical properties that may not hold in more general settings $[29,30]$. Since metabolic exchanges are central to most applications [60,62, 81], we tested the robustness of our results by adding cross-feeding into our model. This was accomplished by specifying the "metabolic leakage matrix" that linked the consumption of each resource to the production of another resource. A certain fraction, $l$, of the produced resources was allowed to "leak" into the environment, where it could be consumed by all species [32].

First, we checked whether our results still hold when there is a nontrivial amount of leakage. This was indeed the case, and landscape ruggedness was predictive of the search efficacy; see Fig. S4.Then, we examined how leakage affected the community, landscape ruggedness, and search efficacy. Leakage tended to increase the number of surviving species and the diversity of the steady-state communities (Fig. 8A). However, it had no systematic affect on any of the ruggedness measures or search efficacy (Fig. 8A,C). Importantly, when we pooled the simulations across all leakage levels, we still found a strong negative relationship between landscape ruggedness and the 

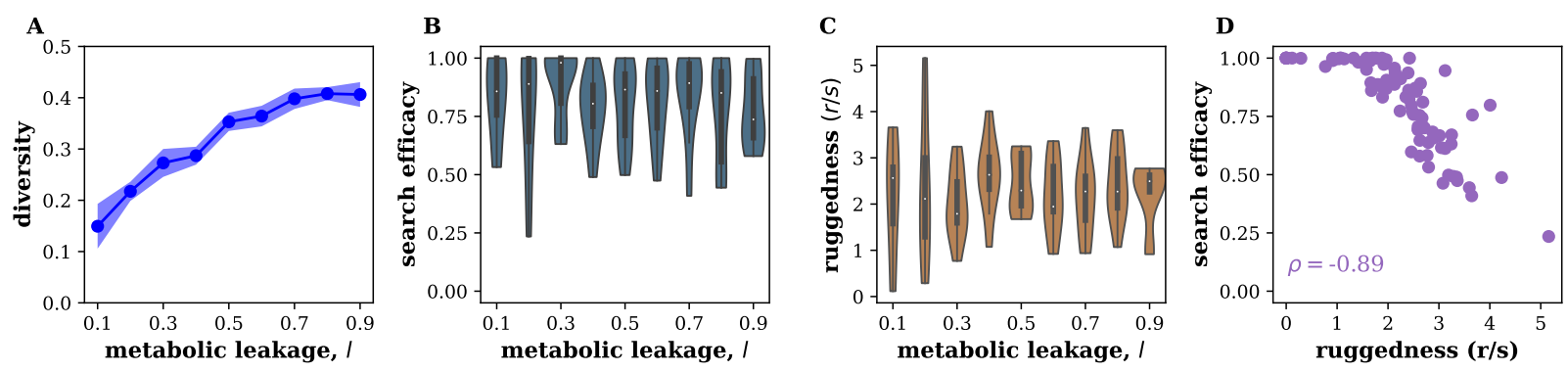

Figure 8: Ruggedness is predictive of search efficacy in models with cross-feeding. Crossfeeding altered the composition of microbial communities (A), but had no systematic effect on the success of the heuristic search (B) or ruggedness (C). Importantly, the search efficacy and ruggedness remained strongly anti-correlated in communities with cross-feeding (D). Simulated species pools had $S=12$ species that leaked a fraction $l$ of the resources they consumed as consumable byproducts. Only one out of 12 modeled resources was supplied externally; the rest were present only due to metabolic leakage. Each species was able to consume 6 resources. Ruggedness was quantified by $r / s$. Community function used in panels B, C, D is the abundance of a focal species. See Methods for further details.

success of the search (Fig. 8D).

The choice of the search protocol also had no major effect on our conclusions. We considered three variations of the simple gradient-ascent considered so far. The first variation was a simple improvement: instead of starting from a single community, the search commenced from the best of $q$ randomly chosen communities. This procedure helped reduce variability of the search length and avoid really poor outcomes due to an unfortunate starting point. We denote this search protocol 'one-step reassembly' as communities at each iteration need to be assembled from scratch using some of the $S$ species.

We also considered two other protocols that mirrored recently proposed community selection methods [82]. These protocols modified the existing community instead of reassembling it from isolates. While adding another species to an existing community is straightforward, removing a species is not. A typical solution of this problem is to perform a strong dilution such that the one or more species become extinct due to the randomness of the dilution process. Our second search protocol was to start from the best of the $q$ communities and then dilute it to create $q$ new communities and $q-1$ of these new communities are diluted further to promote stochastic extinctions. Then then best community is selected for the next iteration. The third search protocol was the same as the second, except a different random species was added to each of the $q-1$ strongly diluted communities. We denote these two dilution-based search protocols as 'bottleneck' and 'addition + bottleneck' respectively.

While the dilution protocols could be appealing, it is worth noting that their performance strongly depended on the dilution factors, which needed to be carefully adjusted for optimal performance. Outside of this narrow range, either too many species went extinct or all species survived (SI Fig. S5). For each protocol, we chose the dilution rate that yielded the best performance. In other words, our results are for the best-case scenario. 


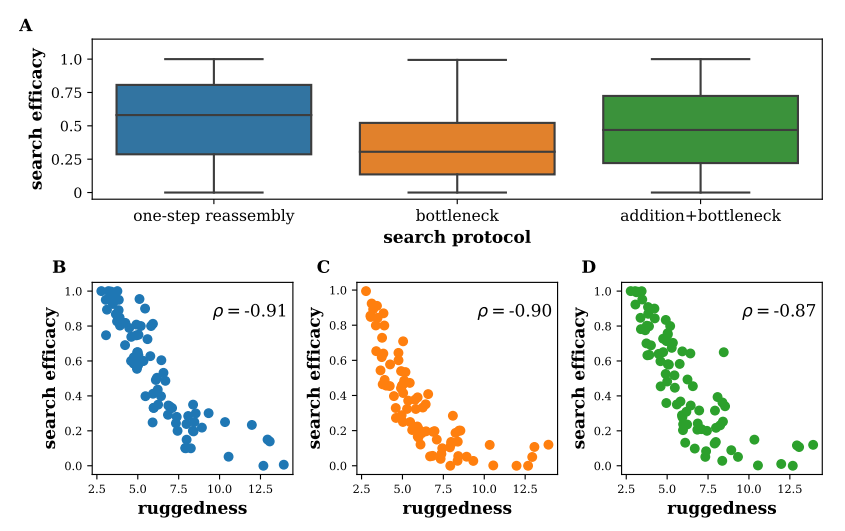

Figure 9: Ruggedness is informative for a variety of search protocols (A) shows the search efficacy of three different community optimization protocols. Despite differences in the performance of different protocols, ruggedness remained informative of search success across protocols (panels B, C, D). Community function was pair-productivity. All searches started from the best of $q=13$ communities. Dilution rate in the dilution-based protocols was adjusted for the highest performance. Simulated communities were the same as in Fig. 8.

The search efficacy differed substantially among protocols. The 'one-step reassembly' and 'addition + bottleneck' performed best in our tests. Importantly, the difference in performance did not affect the relationship between landscape ruggedness and search efficacy (Fig. 9B,C,D).

\section{Ruggedness of an experimental landscape}

We also were able to examine the relationship between landscape ruggedness and search efficacy in an experimental data set. Unfortunately, we found only a single study that had a number of complete ecological landscapes. Langenheder, et. al. [26] measured the metabolic activity of a community of six soil microbes grown on seven different carbon sources in all possible species combinations (Fig. 10).

To check whether these landscape are similar to the ones from consumer-resource models, we determined how well they can be fitted by models of varying complexity (compare to Fig. 5). For all seven experimental landscapes, we found that the accuracy of the fit dramatically improved the complexity of the model and that the quantitative metrics of ruggedness were comparable to those in simulated consumer-resource models (Fig. 10B).

Encouraged by these observations, we computed landscape ruggedness and estimated the performance of the heuristic search. The results are shown in Fig. 10A. In agreement with our expectations, we found that the ruggedness and efficacy were anti-correlated (Spearman's correlation coefficient, $\rho=-0.46)$, but this anti-correlation did not reach statistical significance $(p=0.29)$. The lack of significance could be attributed in part due to the small size of the data set and in part due to the choice of the species and carbon sources. Because of the rather simple metabolic interactions in these communities, the community landscapes were quite smooth and the search efficacy was quite high. Notably, the search efficacy was the highest on the least rugged landscape (xylose), and the search efficacy was perfectly anti-correlated with ruggedness on the three pure carbon sources (Spearman's correlation coefficient, $\rho=-1, p=0.0$ ). 

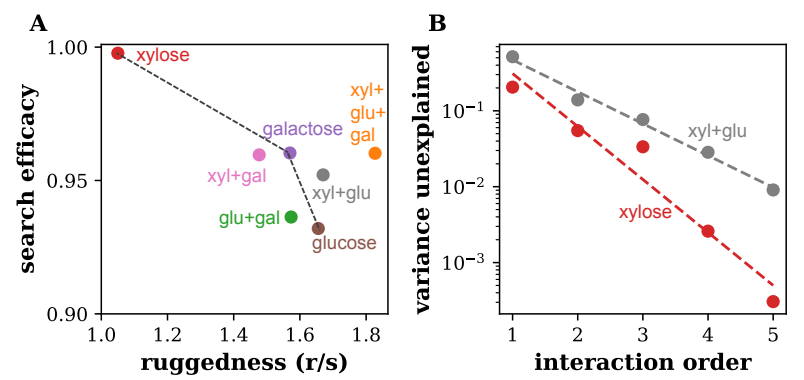

Figure 10: Ruggedness of experimental landscapes (A) shows the ruggedness and search efficacy in the seven environments studied in Ref. [26] labeled by the carbon sources used. There is an apparent anticorrelation between the efficacy and ruggedness, but it does not reach statistical significance $(p=0.29)$. Note that the range of ruggedness and search efficacy is quite narrow presumably because of the overlap in the carbon sources in different environments and the choice of species that grow well on these sugars. Nevertheless, the search efficacy is perfectly anti-correlated with ruggedness on the three pure carbon sources (connected by dashed line) and highest on the least rugged landscape (xylose). (B) The decrease in the variance unexplained with increasing model complexity shows behavior similar to that of the simulated landscapes in Fig. 5. Dashed lines in panel B show a linear fit on the semi-logarithmic plot.

\section{Discussion}

Many problems in biotechnology can be solved by a well-chosen microbial community. However, designing communities is a challenging and multifaceted process. At the very least, it involves identifying promising candidate species and then sifting through the combination of these species to find a productive and stable community. Since an exhaustive assessment of all possible species combinations is rarely possible, an efficient search protocol is key to the design of microbial communities.

A number of such search protocols have been proposed so far $[14,15,83,84]$. All of these protocols share the idea of selecting the best performing communities at each stage of the search. However, many of them failed to improve on the best community in the starting pool because they did not modify the selected communities between search stages [82]. Search protocols that modify the selected community at each search stage should fare better. Protocols based on reassembling the community at each stage, like the ones examined here, are a promising way of systematically exploring the space of possible communities.

To uncover the factors controlling the success of search protocols, we introduced the concept of a community function landscape that describes how the community function changes with community composition, in the context of community design. We focused on ecosystems in which the population comes to a unique steady state that is fully determined by the presence-absence of microbes in the starting community. Although such simple dynamics appear to be quite common, there are well-known exceptions (Table 1). Often, these special cases occur only in a limited region of the parameter space and, at a practical level, can be ignored. This simplification allowed us to rigorously define the community function landscape and propose simple measures to characterize its structure. 
We found that the success of a heuristic search largely depends on the metrics of landscape ruggedness. Three ruggedness metrics stood out as the most useful: $r / s, 1-Z_{\mathrm{nn}}$, and $F_{\text {neut }}$. The roughnessslope metric, $r / s$, was the most predictive in our studies, and estimates from limited data remained highly informative of search. The fraction of neutral directions, $F_{\text {neut }}$, was also predictive and easy to estimate, but had an important downside: It required a quantitative threshold for distinguishing neutral directions, which cannot be determined without additional experiments. In practice, the best metric may vary depending on the nature of the ecosystem and experimental constraints.

We also identified how landscape ruggedness depends on the ecology of the microbial community. Our simulations showed that dense networks of metabolic interactions increase the ruggedness of the landscape and reduce the search efficacy. Thus, it could be important to choose the species and environmental variables in a manner that minimizes unnecessary interactions.

While this study relied heavily on consumer-resource models, we expect that our conclusions hold more generally. Indeed, all of our results remained unchanged when various levels of cross-feeding was incorporated in the model. The analysis of experimental landscapes corroborated our conclusions as well.

Ecological landscapes are very versatile because they can be inferred from both simulations and experiments and can even be compared across different ecosystems. Furthermore, landscapes can be reconstructed simply by measuring the community function across different species combinations. In contrast, alternative optimization approaches require sequencing to infer microbial abundances and a mathematical model to predict desired community composition [68, 85-87] or focus on communities with just two species [88-90]. Ecological landscapes may also facilitate the diffusion of knowledge from other disciplines. One such example is the idea of community coalescence that is analogous to recombination in population genetics [91]. In sum, the versatility of ecological landscapes and the practicality of ruggedness metrics can make them a valuable tool for bioengineering applications. 


\section{Methods}

\section{Consumer-resource models with and without cross-feeding}

We simulated a consumer-resource model where species competed for $M_{\text {total }}$ resources, which were supplied externally $[27,92]$. A species $i$ had abundance $n_{i}$, growth rate $g_{i}$, and maintenance cost $m_{i}$. A resource $\alpha$ had concentration $R_{\alpha}$ and quality $w_{\alpha}$. Resource supply resembled a chemostat with supply point $R_{\alpha}^{0}$ and dilution rate $\tau^{-1}$. Species differed in their preferences for the various resources, encoded in the consumption preference matrix $C$. A species could consume $M_{\text {consumed }}$ randomly chosen resources out of the $M_{\text {total }}$ supplied resources. The strength of this preference was randomly drawn from a gamma distribution. The equations governing the dynamics of the species and resources are:

$$
\begin{aligned}
\frac{d n_{i}}{d t} & =g_{i} n_{i}\left[\sum_{\alpha=1}^{M} w_{\alpha} \mu\left(C_{i \alpha} R_{\alpha}\right)-m_{i}\right], \\
\frac{d R_{\alpha}}{d t} & =\tau^{-1}\left(R_{\alpha}^{0}-R_{\alpha}\right)-\sum_{i=1}^{S} n_{i} \mu\left(C_{i \alpha} R_{\alpha}\right),
\end{aligned}
$$

where $\mu$ is a function describing resource uptake rates. For the consumer resource model without cross-feeding, we assumed a simple linear form for the uptake.

Simulations were carried out in python using custom code and a modified version of the community simulator package [64]. Obtaining the steady state via direct numerical integration of the ODEs is computationally expensive as the number of species combinations grows exponentially with pool size. Therefore, we utilized a recently discovered mapping between ecological models and convex optimization to calculate the steady state quickly $[64,93]$. Species with an abundance below $10^{-6}$ were set to be extinct.

To incorporate the secretion and uptake of metabolites by microbes, we examined the microbial consumer-resource model with cross-feeding [32]. In the cross-feeding model, each species leaks a fraction, $l$, of resources it consumes in the form of metabolic byproducts. The composition of these byproducts is specified by the metabolic leakage matrix $\mathcal{L}$, with matrix element $\mathcal{L}_{\alpha \beta}$ specifying the amount of resource $\beta$ leaked when the species consumes resource $\alpha$. This leakage is weighted by the ratio of resource qualities $w_{\beta} / w_{\alpha}$ so that energy-poor resources cannot produce a disproportionate amount of energy-rich resources. Each row of the leakage matrix sums to one. The leakage matrix and resource qualities were independent of species identity to respect potential universal stoichiometric constraints on species metabolism. The dynamical equations of the model with cross-feeding are:

$$
\begin{aligned}
\frac{d n_{i}}{d t} & =g_{i} n_{i}\left[(1-l) \sum_{\alpha=1}^{M} w_{\alpha} \mu\left(C_{i \alpha} R_{\alpha}\right)-m_{i}\right] \\
\frac{d R_{\alpha}}{d t} & =\tau^{-1}\left(R_{\alpha}^{0}-R_{\alpha}\right)-\sum_{i=1}^{S} n_{i} \mu\left(C_{i \alpha} R_{\alpha}\right)+l \sum_{i=1}^{S} \sum_{\beta=1}^{M} n_{i} \mathcal{L}_{\alpha \beta} \frac{w_{\beta}}{w_{\alpha}} \mu\left(c_{i \beta} R_{\beta}\right) .
\end{aligned}
$$

Here, resource uptake rates were assumed to be of Monod form, $\mu(x)=x /\left(1+\frac{x}{K_{\alpha}}\right)$. We simulated the system of ODEs explicitly using the community simulator package to obtain steady-state abundances. Since direct simulation of the ODEs is computationally expensive, we simulated smaller 
candidate species pools with $S=12$. Simulations reached steady state if the root mean square of the logarithmic species growth rates rates fell below a threshold, i.e., RMS $\left(\frac{1}{n_{i}} \frac{d n_{i}}{d t}\right)<10^{-3}$. We imposed an abundance cutoff at periodic intervals during the numerical integration to hasten the extinction of species. We verified that the extinct species could not have survived in the community by simulating a re-invasion attempt of the steady-state community.

\section{Simulation parameters}

In simulations without cross-feeding, we simulated candidate species pools with $S$ species and supplied $M_{\text {total }}$ resources. All species within a candidate species pool consumed a fixed number of resources, $M_{\text {consumed }}$, chosen randomly for each species. The strength of these non-zero consumption preferences were chosen from a gamma distribution with parameters $(k, \theta)=(10,0.1)$. Growth rates $g_{i}$ and death rates $m_{i}$ were sampled from normal distributions with mean 1.0 and variance 0.1 (truncated to ensure positivity). Resource quality $w_{\alpha}$ and chemostat fixed point $R_{\alpha}^{0}$ were sampled from gamma distributions parameterized by $(k, \theta)=(10,0.1)$ and $(k, \theta)=(10,2)$; chemostat dilution rate $\tau^{-1}$ was set to 1 .

In these simulations, we varied $M_{\text {consumed }}$ from 1 resource to $M_{\text {total }}$ resources across different candidate pools. We simulated ten pools at each value of $M_{\text {consumed }}$. We examined candidate pools of varying sizes: Figure 2 corresponds to simulations for $\left(S, M_{\text {total }}\right)=\{(4,4),(8,8),(12,12),(16,16)\}$. Figures 4, 5, 6, 7 present data from the 16 species candidate pools. We also simulated and analyzed results for $\left(S, M_{\text {total }}\right)=\{(8,4),(12,6),(16,8)\}$ and $\left(S, M_{\text {total }}\right)=\{(8,16),(12,24),(16,32)\}$ to verify that our results are robust to the number of supplied resources.

In simulations with cross-feeding (Figs. 8, 9), we simulated $S=12$ candidate species and $M_{\text {total }}=12$ resources. Only a single resource, $R_{0}$, was supplied externally. The resource was supplied in sufficient amounts (with a chemostat fixed point $R_{0}^{0}=240$ ) that many species were able to survive by feeding on the byproducts of the consumers of the supplied resource. Species leaked a fraction $l$ of the consumed resources in forms specified by the leakage matrix. Each column of the leakage matrix was sampled from a Dirichlet distribution with parameter 0.5. Using the Dirichlet distribution guaranteed that each column summed to one. The Monod form for resource uptake had parameter $K_{\alpha}=20$. The chemostat dilution rate was set to 0.1. All other parameters: growth rates $g_{i}$, death rates $m_{i}$, resource quality $w_{\alpha}$, and strength of consumption preferences $C_{i \alpha}$, were sampled as in the consumer-resource model.

We performed three types of simulations with the cross-feeding model-examining the effect of the extent of cross-feeding, environmental complexity, and niche overlap. Parameters were chosen as below:

1. To examine the effect of the extent of cross-feeding, we varied the metabolic leakage fraction $l$ from 0.1 to 0.9 across species pools. The number of resources consumed by each species, $M_{\text {consumed }}$, was 6 . To ensure that at least one species in every candidate pool could consume the supplied resource, we constrained species 0 to always consume the sole supplied resource $R_{0}$ and species 1 to never consume $R_{0}$. (Both species could still only consume 6 resources in total). The abundance cutoff was $10^{-6}$. These simulations are analyzed in Figs. 8, 9.

2. To examine the impact of environmental complexity, we varied the number of resources supplied, $R_{\text {supplied }}$, from 1 to 12 across species pools. We fixed $l=0.5$ and $M_{\text {consumed }}=6$. We fixed the 
total supply flux of resources by setting the individual resource supply points to $240 / R_{\text {supplied }}$. The abundance cutoff was $10^{-3}$. Results are shown in SI Fig. S6. Similar results were obtained when we allowed the total resource supply flux to increase with the number of supplied resources (each individual resource had supply point at 240).

3. To examine effect of niche overlap, we vary the number of resources consumed from 1 (extreme specialists) to 12 (extreme generalists). The metabolic leakage fraction was fixed at $l=0.5$. We constrained species 0 to always consume the sole supplied resource $R_{0}$, to ensure that at least one species ate the supplied resource. The abundance cutoff was $10^{-3}$. Results are shown in SI Fig. S4

For each set of parameters, we generated 10 candidate pools differing only by the random sampling.

\section{Characterizing the map to steady-state abundances $\vec{N}(\vec{\sigma})$}

Using the simulation models parameterized as above, we simulated all possible combinations of species in the candidate species pools till they reached steady-state. To characterize the assembly map $\vec{N}(\vec{\sigma})$, we quantified how well observed abundance of each species is explained by adding independent contributions from other community members. Therefore, we fit the steady-state abundance of a species $i, N_{i}(\vec{\sigma})$, using the model

$$
N_{i}(\vec{\sigma})= \begin{cases}\sum_{j=1}^{S} A_{i j} \sigma_{j}+\epsilon, & \text { if } \sigma_{i}=1 \\ 0 & \text { if } \sigma_{i}=0\end{cases}
$$

where $\sigma_{i}$ and $\sigma_{j}$ denote the presence-absence of species $i$ and $j, A$ is the matrix of effective interactions, and $\epsilon$ is the model error. Note that the model can predict negative abundances, which we set to zero. This truncation did not affect our results.

For each species $i$, we perform a least squares linear regression to infer the corresponding row (row $i)$ of matrix $A$ using data from communities where the species was initially present $\left(\sigma_{i}=1\right)$.

The abundance predicted by the model, $\hat{N}_{i}(\vec{\sigma})$, is given by

$$
\hat{N}_{i}(\vec{\sigma})= \begin{cases}\sum_{j=1}^{S} A_{i j} \sigma_{j} & \text { if } \sigma_{i}=1 \\ 0 & \text { if } \sigma_{i}=0 .\end{cases}
$$

We record the fraction of the variance explained (equivalent to the coefficient of determination) by the model, and repeat the regression for each of the $S$ species. The average variance explained across all $S$ species, $\overline{R^{2}}$, is a measure of the linearity of the abundance landscape. Results are presented in Fig. 2.

\section{Community function landscapes}

We computed the community function of interest, $\mathcal{F}$, from the steady-state species abundances. The three forms of community functions we analyzed are:

1. Shannon Diversity was measured as

$$
\text { diversity }=\frac{1}{\ln S} \sum_{i=1}^{S} p_{i} \ln p_{i}
$$


where $p_{i}$ is the relative abundance of species $i$.

2. Pair Productivity, mediated by a pair of species $(A, B)$, was measured as the product of their abundances,

$$
\text { pair productivity }=N_{A} N_{B}
$$

This approximates a scenario where a useful product is synthesized by the combined activity of two species.

3. Focal abundance. The abundance of a focal species of interest was given by $N_{\text {focal }}$. Since this function makes sense only if the species was present in the initial community, we restricted our analyses to the subset of the complete landscape where the focal species was initially present. This subset resembles a landscape made by $S-1$ species, with $2^{S-1}$ species combinations.

\section{Landscape ruggedness}

We used three ruggedness measures to characterize the community function landscapes:

1. The roughness-slope ratio $r / s$ is given by the ratio of roughness $r$ to slope $s$ of the landscape. The roughness, $r$, measures the root-mean-squared residual of the linear fit. It is obtained by fitting the community function $\mathcal{F}$ with the linear model, $\hat{\mathcal{F}}(\vec{\sigma})=c+\sum_{i=1}^{S} a_{i} \sigma_{i}$, via least squares regression. $r$ is given by

$$
r=\sqrt{\frac{1}{\|\vec{\sigma}\|} \sum_{\vec{\sigma}}(\mathcal{F}(\vec{\sigma})-\hat{\mathcal{F}}(\vec{\sigma}))^{2}},
$$

where $\|\vec{\sigma}\|$ is the number of points on the landscape.

The slope, $s$, measures the magnitude of change in $\mathcal{F}$ when a species is added or removed. It is given by

$$
s=\frac{1}{S} \sum_{i=1}^{S}\left|a_{i}\right|
$$

2. $1-Z_{n n}$, where $Z_{\mathrm{nn}}$ is nearest neighbor correlation on the landscape. $Z_{\mathrm{nn}}$ is defined as :

$$
Z_{n n}=\frac{\frac{1}{\left\|\vec{\sigma}^{n n}\right\|} \sum_{\vec{\sigma}, \vec{\sigma}^{n n}} \mathcal{F}(\vec{\sigma}) \mathcal{F}\left(\vec{\sigma}^{n n}\right)-\overline{\mathcal{F}}^{2}}{\sum_{\vec{\sigma}}(\mathcal{F}(\vec{\sigma})-\overline{\mathcal{F}})^{2}} .
$$

$\vec{\sigma}^{n n}$ refers to the nearest neighbors of $\vec{\sigma}$ on the landscape, i.e., communities obtained by adding or removing a single species, and $\left\|\vec{\sigma}^{n n}\right\|$ is the number of nearest neighbors.

3. The fraction of quasi-neutral directions $F_{\text {neut }}$ is a third measure of ruggedness. It was measured by computing the fraction of nearest neighbors on the landscape with function values that differed by less than a defined threshold $\left(10^{-4}\right)$. 


\section{Variance unexplained by higher-order models}

We fit ecological landscapes of community function $\mathcal{F}(\vec{\sigma})$ incorporating higher-order species interactions using the following class of models:

$$
\hat{\mathcal{F}}^{(k)}(\vec{\sigma})=a^{(0)}+\sum_{i=1}^{S} a_{i}^{(1)} \sigma_{i},+\sum_{i=1}^{S} \sum_{j=1}^{i-1} a_{i j}^{(2)} \sigma_{i} \sigma_{j}+\ldots \mathcal{O}\left(a^{(k)}\right)+\epsilon(\vec{\sigma}),
$$

where the fit coefficients $a^{(0)}, a^{(1)}, \ldots$ defined up to order $k$ are obtained by least squares regression minimizing $\left(\hat{\mathcal{F}}^{(k)}(\vec{\sigma})-\mathcal{F}(\vec{\sigma})\right)^{2}$. (Note that we can still use linear regression because the models are linear in the interaction coefficients.)

The fraction of variance unexplained by the model of order $k, U^{(k)}$, is given by

$$
U^{(k)}=1-\frac{\sum_{\vec{\sigma}}\left(\hat{\mathcal{F}}^{(k)}(\vec{\sigma})-\overline{\mathcal{F}}\right)^{2}}{\sum_{\vec{\sigma}}(\mathcal{F}(\vec{\sigma})-\overline{\mathcal{F}})^{2}}
$$

where $\overline{\mathcal{F}}$ is the mean function; the numerator and denominator are the explained sum of squares and total sum of squares respectively. The number of higher order terms grows exponentially with the number of species, and so inverting matrices for linear regression became computationally expensive for large landscapes. Therefore, we used an alternative method to calculate the the fraction of variance unexplained for large landscapes based on Walsh decomposition (see SI).

We imposed a threshold of $10^{-6}$ in the fraction of variance unexplained when plotting and fitting data in Fig. 5 to be robust to numerical artifacts.

\section{Heuristic search protocols}

We simulated community optimization attempts via a number of different search protocols.

\section{Heuristic gradient-ascent search and one-step reassembly}

The gradient-ascent search proceeds as follows:

1. Choose a starting point on the landscape $A$, with initial composition $\vec{\sigma}^{A}$ and community function $\mathcal{F}\left(\vec{\sigma}^{A}\right)$.

2. Evaluate the relative change in steady-state community function at all points a single step away on the landscape, i.e, communities obtained by adding or removing a single species to the initial composition at $A$.

$$
\Delta^{B}=\frac{\mathcal{F}\left(\vec{\sigma}^{B}\right)-\mathcal{F}\left(\vec{\sigma}^{A}\right)}{\mathcal{F}\left(\vec{\sigma}^{A}\right)}, \forall B:\left|\vec{\sigma}^{B}-\vec{\sigma}^{A}\right|=1
$$

3. If the community function increased for any of the neighboring communities $\left(\Delta^{B}>10^{-4}\right)$, then we choose the neighboring point with highest community function and repeat from step 2 with this chosen community as $A$.

4. If the community function did not increase at any neighboring community, the search terminates. The current community is the community found by the search.

In Fig. 9, we study a modified protocol, 'one-step reassembly' where search started from the best point out of $q$ randomly chosen communities. We also simulated a version of gradient-ascent search 
where search proceeded along the first direction with increasing community function (instead of the direction with maximal increase in community function). Similar results were obtained for this version and we omit discussion of these results for brevity.

\section{'bottleneck' protocol}

The 'bottleneck' protocol proceeds as follows:

1. Grow $q$ random communities till steady state. Choose the community with highest function as the starting community.

2. $q$ replicates of the chosen community are generated.

3. $q-1$ replicates are passaged via severe dilution shocks to generate stochastic species extinctions. One replicate is left unperturbed.

4. The $q$ communities are grown to steady state, and the community with the highest function is chosen.

5. Repeat steps $2-4$ for a fixed number of iterations. The best community among the $q$ communities in the last iteration is the community found by the search protocol.

To simulate a dilution shock, the number of cells in the community $n_{\text {cells }}$ was first calculated by dividing by a fixed biomass conversion factor of $10^{-3}$. The number of cells in the community after dilution by factor $D$ was sampled from a Poisson distribution with mean $D n_{\text {cells. }}$ The number of cells of each species was then obtained by multinomial sampling with probabilities proportional to the biomass of each species.

\section{'addition + bottleneck' protocol}

The 'addition + bottleneck' protocol closely follows the 'bottleneck' protocol above, with a single modification. In Step 3, after $q-1$ replicates are subject to severe dilution shocks, a different randomly chosen species is added to each of these replicates. The $q^{\text {th }}$ replicate is still left unperturbed.

We simulated both dilution-based protocols ('bottleneck' and 'addition + bottleneck' ) for a range of dilution factors. The two strategies performed best at an intermediate dilution, where dilution stochastically eliminates a few species from the community, but not too many species (Fig. S5). We report the results for the dilution factor that had the highest search efficacy in the main text (Fig. 9).

To ensure a fair comparison between the dilution-based protocols and one-step reassembly, we fixed the number of replicates to $q=S+1$ and the number of iterations to $S / 2$ in the dilution-based protocols, and started one-step reassembly from the best of $q$ random communities in Fig. 9. This ensured that the number of experiments in all strategies were similar.

Search efficacy of all search protocols was evaluated as the ratio between the community function at the end point of the search and the best community function on the landscape. We report search efficacy averaged over all possible starting points on the landscape in figures on the simple gradientascent search protocol. In Fig. 9, which compared the dilution-based protocols and 'one-step reassembly' we report the search efficacy averaged over 10 independent searches on each landscape. 


\section{Sampling ecological landscapes}

We estimated ruggedness measures from appropriately sampled subsets of the community function landscapes. To estimate $r / s$, we randomly sampled a fraction, $f$, of the possible $2^{S}$ communities. The linear model was fit on the sampled data points to estimate $r / s$. Since the model has $S+1$ parameters, we need to sample at least $S+1$ points. To estimate $1-Z_{n n}$ and $F_{\text {neut }}$, we sampled a fraction $f$ of all communities by choosing half of the communities randomly and generating one random nearest neighbor for each of them. $1-Z_{n n}$ and $F_{\text {neut }}$ were then calculated from the sampled points.

\section{Analysis of experimental data}

The experiments by Langenheder, et. al. measured the time series of cumulative metabolic activity, through the intensity of a dye, for each community and environment [26]. We obtained the rate of metabolic activity from the data via a linear fit of the metabolic activity over the last six time points. The communities had reached a steady state in metabolic activity, as evidenced by the high $R^{2}$ of the linear fits (SI Fig. S7). We analyzed the landscape formed by the steady-state metabolic rate in Fig. 10. We imposed a threshold in the fraction of variance unexplained $\left(10^{-4}\right)$ when plotting and fitting data in Fig. 10B.

\section{Acknowledgments}

The authors would like to thank Pankaj Mehta, Gabriel Birzu, Shreya Arya, and James O'Dwyer for helpful discussions and comments, and Silke Langenheder for sharing experimental data. This work was supported by the Simons Foundation Grant \#409704, Cottrell Scholar Award \#24010, and by NIGMS grant \#1R01GM138530-01 to K.S.K. Simulations were carried out on the Boston University Shared Computing Cluster.

\section{References}

[1] Paerl, H. \& Pinckney, J. A mini-review of microbial consortia: Their roles in aquatic production and biogeochemical cycling. Microbial Ecology 31 (1996). URL http://link.springer.com/10.1007/BF00171569.

[2] Falkowski, P. G., Fenchel, T. \& Delong, E. F. The Microbial Engines That Drive Earth's Biogeochemical Cycles. Science 320, 1034-1039 (2008). URL https://www.sciencemag.org/lookup/doi/10.1126/science.1153213.

[3] Flint, H. J., Scott, K. P., Duncan, S. H., Louis, P. \& Forano, E. Microbial degradation of complex carbohydrates in the gut. Gut Microbes 3, 289-306 (2012). URL http://www.tandfonline.com/doi/abs/10.4161/gmic.19897.

[4] Brenner, K., You, L. \& Arnold, F. H. Engineering microbial consortia: a new frontier in synthetic biology. Trends in Biotechnology 26, 483-489 (2008). URL https://linkinghub.elsevier.com/retrieve/pii/S0167779908001716.

[5] Olle, B. Medicines from microbiota. Nature Biotechnology 31, 309-315 (2013). URL http://www . nature.com/articles/nbt. 2548. 
[6] Lindemann, S. R. et al. Engineering microbial consortia for controllable outputs. The ISME Journal 10, 2077-2084 (2016). URL http://www. nature.com/articles/ismej 201626.

[7] Minty, J. J. et al. Design and characterization of synthetic fungal-bacterial consortia for direct production of isobutanol from cellulosic biomass. Proceedings of the National Academy of Sciences 110, 14592-14597 (2013). URL http://www.pnas.org/lookup/doi/10.1073/pnas.1218447110.

[8] Tanoue, T. et al. A defined commensal consortium elicits CD8 T cells and anti-cancer immunity. Nature 565, 600-605 (2019). URL http://www. nature.com/articles/s41586-019-0878-z.

[9] Buffie, C. G. et al. Precision microbiome reconstitution restores bile acid mediated resistance to Clostridium difficile. Nature 517, 205-208 (2015). URL http://www . nature.com/articles/nature13828.

[10] $\mathrm{Hu}$, J. et al. Design and composition of synthetic fungal-bacterial microbial consortia that improve lignocellulolytic enzyme activity. Bioresource Technology 227, 247-255 (2017). URL https://linkinghub.elsevier.com/retrieve/pii/S096085241631728X.

[11] Stein, R. R. et al. Computer-guided design of optimal microbial consortia for immune system modulation. eLife 7 (2018). URL https://elifesciences.org/articles/30916.

[12] Kehe, J. et al. Massively parallel screening of synthetic microbial communities. Proceedings of the National Academy of Sciences 116, 12804-12809 (2019). URL http://www .pnas.org/lookup/doi/10.1073/pnas.1900102116.

[13] Villa, M. M. et al. High-throughput isolation and culture of human gut bacteria with droplet microfluidics. bioRxiv (2019). URL http://biorxiv.org/lookup/doi/10.1101/630822.

[14] Swenson, W., Arendt, J. \& Wilson, D. S. Artificial selection of microbial ecosystems for 3-chloroaniline biodegradation. Environmental Microbiology 2, 564-571 (2000). URL http://doi.wiley.com/10.1046/j.1462-2920.2000.00140.x.

[15] Chang, C.-Y., Osborne, M. L., Bajic, D. \& Sanchez, A. Artificially selecting microbial communities using propagule strategies. preprint, Evolutionary Biology (2020). URL http://biorxiv.org/lookup/doi/10.1101/2020.05.01.066282.

[16] Eng, A. \& Borenstein, E. Microbial community design: methods, applications, and opportunities. Current Opinion in Biotechnology 58, 117-128 (2019). URL https://linkinghub.elsevier.com/retrieve/pii/S0958166918301927.

[17] Chen, Y., Lin, C.-J., Jones, G., Fu, S. \& Zhan, H. Enhancing biodegradation of wastewater by microbial consortia with fractional factorial design. Journal of Hazardous Materials 171, 948953 (2009). URL https://linkinghub.elsevier.com/retrieve/pii/S0304389409010255.

[18] Tsoi, R., Dai, Z. \& You, L. Emerging strategies for engineering microbial communities. Biotechnology Advances 37, 107372 (2019). URL https://linkinghub.elsevier.com/retrieve/pii/S0734975019300473.

[19] Hsu, R. H. et al. Microbial Interaction Network Inference in Microfluidic Droplets. Cell Systems 9, 229-242.e4 (2019). URL https://linkinghub.elsevier.com/retrieve/pii/S2405471219302315. 
[20] Zanakis, S. H. \& Evans, J. R. Heuristic "Optimization": Why, When, and How to Use It. Interfaces 11, 84-91 (1981). URL http://pubsonline.informs.org/doi/abs/10.1287/inte.11.5.84.

[21] Rardin, R. L. \& Uzsoy, R. Experimental Evaluation of Heuristic Optimization Algorithms: A Tutorial. Journal of Heuristics 261-304 (2001).

[22] Marsland III, R., Cui, W. \& Mehta, P. A minimal model for microbial biodiversity can reproduce experimentally observed ecological patterns. arXiv:1904.12914 [physics, q-bio] (2019). URL http://arxiv.org/abs/1904.12914. ArXiv: 1904.12914.

[23] Ho, P.-Y., Good, B. \& Huang, K. C. Competition for fluctuating resources reproduces statistics of species abundance over time across wide-ranging microbiotas. preprint, Ecology (2021). URL http://biorxiv.org/lookup/doi/10.1101/2021.05.13.444061.

[24] Gowda, K., Ping, D., Mani, M. \& Kuehn, S. A sparse mapping of structure to function in microbial communities. preprint, Ecology (2020). URL http://biorxiv.org/lookup/doi/10.1101/2020.09.29.315713.

[25] Wright, S. The roles of mutation, inbreeding, crossbreeding, and selection in evolution. Proc. VIth Int. Congress of Genetics 1, 356-366 (1932).

[26] Langenheder, S., Bulling, M. T., Solan, M. \& Prosser, J. I. Bacterial Biodiversity-Ecosystem Functioning Relations Are Modified by Environmental Complexity. PLoS ONE 5, e10834 (2010). URL https://dx.plos.org/10.1371/journal . pone.0010834.

[27] MacArthur, R. Species packing and competitive equilibrium for many species. Theoretical Population Biology 1, 1-11 (1970). URL https://linkinghub.elsevier. com/retrieve/pii/0040580970900390.

[28] Armstrong, R. A. \& McGehee, R. Competitive Exclusion. The American Naturalist 115, 151-170 (1980). URL http://www.jstor.org/stable/2460592. Publisher: [University of Chicago Press, American Society of Naturalists].

[29] Tikhonov, M. Community-level cohesion without cooperation. eLife 5, e15747 (2016). URL https://elifesciences.org/articles/15747.

[30] Marsland III, R., Cui, W. \& Mehta, P. The Minimum Environmental Perturbation Principle: A New Perspective on Niche Theory. arXiv:1901.09673 [physics, q-bio] (2019). URL http://arxiv.org/abs/1901.09673. ArXiv: 1901.09673.

[31] Ispolatov, I. \& Doebeli, M. A note on the complexity of evolutionary dynamics in a classic consumer-resource model. Theoretical Ecology (2019). URL http://link.springer.com/10.1007/s12080-019-0427-2.

[32] Marsland, R. et al. Available energy fluxes drive a transition in the diversity, stability, and functional structure of microbial communities. PLOS Computational Biology 15, e1006793 (2019). URL http://dx.plos.org/10.1371/journal.pcbi.1006793.

[33] Butler, S. \& O’Dwyer, J. P. Stability criteria for complex microbial communities. Nature Communications 9 (2018). URL http://www. nature.com/articles/s41467-018-05308-z. 
[34] Roy, F., Biroli, G., Bunin, G. \& Cammarota, C. Numerical implementation of dynamical mean field theory for disordered systems: application to the Lotka-Volterra model of ecosystems. arXiv:1901.10036 [cond-mat, q-bio] (2019). URL http://arxiv.org/abs/1901.10036. ArXiv: 1901.10036.

[35] Kessler, D. A. \& Shnerb, N. M. Generalized model of island biodiversity. Physical Review E 91 (2015). URL https://link.aps.org/doi/10.1103/PhysRevE.91.042705.

[36] Smillie, J. Competitive and Cooperative Tridiagonal Systems of Differential Equations. SIAM Journal on Mathematical Analysis 15, 530-534 (1984). URL http://epubs.siam.org/doi/10.1137/0515040.

[37] Smith, H. L. Competing Subcommunities of Mutualists and a Generalized Kamke Theorem. SIAM Journal on Applied Mathematics 46, 856-874 (1986). URL http://epubs.siam.org/doi/10.1137/0146052.

[38] Bunin, G. Directionality and community-level selection. bioRxiv (2018). URL http://biorxiv.org/lookup/doi/10.1101/484576.

[39] Gibson, T. E., Bashan, A., Cao, H.-T., Weiss, S. T. \& Liu, Y.-Y. On the Origins and Control of Community Types in the Human Microbiome. PLOS Computational Biology 12, e1004688 (2016). URL https://dx.plos.org/10.1371/journal.pcbi.1004688.

[40] Vanwonterghem, I. et al. Deterministic processes guide long-term synchronised population dynamics in replicate anaerobic digesters. The ISME Journal 8, 2015-2028 (2014). URL http://www. nature.com/articles/ismej201450.

[41] Aranda-Díaz, A. et al. High-throughput cultivation of stable, diverse, fecal-derived microbial communities to model the intestinal microbiota. preprint, Microbiology (2020). URL http://biorxiv.org/lookup/doi/10.1101/2020.07.06.190181.

[42] Lucas, R., Kuchenbuch, A., Fetzer, I., Harms, H. \& Kleinsteuber, S. Long-term monitoring reveals stable and remarkably similar microbial communities in parallel full-scale biogas reactors digesting energy crops. FEMS Microbiology Ecology 91 (2015). URL https://academic.oup.com/femsec/article-lookup/doi/10.1093/femsec/fiv004.

[43] Dubinkina, V., Fridman, Y., Pandey, P. P. \& Maslov, S. Multistability and regime shifts in microbial communities explained by competition for essential nutrients. eLife 8 (2019). URL https://elifesciences.org/articles/49720.

[44] Wang, Z. et al. Complementary resource preferences spontaneously emerge in diauxic microbial communities. Nature Communications 12, 6661 (2021). URL https://www . nature.com/articles/s41467-021-27023-y.

[45] Friedman, J., Higgins, L. M. \& Gore, J. Community structure follows simple assembly rules in microbial microcosms. Nature Ecology $\&$ Evolution 1 (2017). URL http://www . nature.com/articles/s41559-017-0109.

[46] Estrela, S. et al. Functional attractors in microbial community assembly. Cell Systems S2405471221003793 (2021). URL https://linkinghub.elsevier.com/retrieve/pii/S2405471221003793. 
[47] Fukami, T., Martijn Bezemer, T., Mortimer, S. R. \& Putten, W. H. Species divergence and trait convergence in experimental plant community assembly. Ecology Letters 8, 1283-1290 (2005). URL http://doi.wiley.com/10.1111/j.1461-0248.2005.00829.x.

[48] Louca, S. et al. High taxonomic variability despite stable functional structure across microbial communities. Nature Ecology \& Evolution 1 (2017). URL http://www.nature.com/articles/s41559-016-0015.

[49] Hubbell, S. P. The unified neutral theory of biodiversity and biogeography. No. 32 in Monographs in population biology (Princeton University Press, Princeton, 2001).

[50] Maslov, S. \& Sneppen, K. Population cycles and species diversity in dynamic Kill-the-Winner model of microbial ecosystems. Scientific Reports 7 (2017). URL http://www . nature.com/articles/srep39642.

[51] Huisman, J. \& Weissing, F. J. BIOLOGICAL CONDITIONS FOR OSCILLATIONS AND CHAOS GENERATED BY MULTISPECIES COMPETITION. Ecology 82, 2682-2695 (2001). URL http://doi .wiley.com/10 .1890/0012-9658(2001)082 [2682:BCFOAC]2.0.CO;2.

[52] Pearce, M., Agarwala, A. \& Fisher, D. S. Stabilization of extensive fine-scale diversity by spatio-temporal chaos. bioRxiv (2019). URL http://biorxiv.org/lookup/doi/10.1101/736215.

[53] Ofiteru, I. D. et al. Combined niche and neutral effects in a microbial wastewater treatment community. Proceedings of the National Academy of Sciences 107, 15345-15350 (2010). URL http://www.pnas.org/cgi/doi/10.1073/pnas.1000604107.

[54] Samuels, C. L. \& Drake, J. A. Divergent perspectives on community convergence. Trends in Ecology \& Evolution 12, 427-432 (1997). URL https://linkinghub.elsevier.com/retrieve/pii/S0169534797011828.

[55] Momeni, B., Xie, L. \& Shou, W. Lotka-Volterra pairwise modeling fails to capture diverse pairwise microbial interactions. eLife 6, e25051 (2017). URL https://elifesciences.org/articles/25051.

[56] O'Dwyer, J. P. Whence Lotka-Volterra?: Conservation laws and integrable systems in ecology. Theoretical Ecology 11, 441-452 (2018). URL http://link. springer.com/10.1007/s12080-018-0377-0.

[57] Hoek, T. A. et al. Resource Availability Modulates the Cooperative and Competitive Nature of a Microbial Cross-Feeding Mutualism. PLOS Biology 14, e1002540 (2016). URL https://dx.plos.org/10.1371/journal.pbio.1002540.

[58] Gopalakrishnappa, C., Gowda, K., Prabhakara, K. \& Kuehn, S. An ensemble approach to the structure-function problem in microbial communities. arXiv:2111.06279 [q-bio] (2021). URL http://arxiv.org/abs/2111.06279. ArXiv: 2111.06279.

[59] Niehaus, L. et al. Microbial coexistence through chemical-mediated interactions. Nature Communications 10, $2052 \quad$ (2019). URL http: //www . nature. com/articles/s41467-019-10062-x. 
[60] D'Souza, G. et al. Ecology and evolution of metabolic cross-feeding interactions in bacteria. Natural Product Reports 35, 455-488 (2018). URL http: //xlink.rsc .org/?DOI=C8NP00009C.

[61] Pacheco, A. R., Moel, M. \& Segrè, D. Costless metabolic secretions as drivers of interspecies interactions in microbial ecosystems. Nature Communications 10 (2019). URL http://www. nature.com/articles/s41467-018-07946-9.

[62] Datta, M. S., Sliwerska, E., Gore, J., Polz, M. F. \& Cordero, O. X. Microbial interactions lead to rapid micro-scale successions on model marine particles. Nature Communications 7, 11965 (2016). URL http://www . nature.com/articles/ncomms11965.

[63] Gralka, M., Szabo, R., Stocker, R. \& Cordero, O. X. Trophic Interactions and the Drivers of Microbial Community Assembly. Current Biology 30, R1176-R1188 (2020). URL https://linkinghub.elsevier.com/retrieve/pii/S0960982220311611.

[64] Marsland, R., Cui, W., Goldford, J. \& Mehta, P. The Community Simulator: A Python package for microbial ecology. bioRxiv (2019). URL http://biorxiv.org/lookup/doi/10.1101/613836.

[65] Hang-Kwang, L. \& Pimm, S. L. The Assembly of Ecological Communities: A Minimalist Approach. The Journal of Animal Ecology 62, 749 (1993). URL https://www.jstor.org/stable/5394?origin=crossref.

[66] Morton, R. D., Law, R., Pimm, S. L. \& Drake, J. A. On Models for Assembling Ecological Communities. Oikos 75, 493 (1996). URL https://www.jstor.org/stable/3545891?origin=crossref.

[67] Bittleston, L. S., Gralka, M., Leventhal, G. E., Mizrahi, I. \& Cordero, O. X. Context-dependent dynamics lead to the assembly of functionally distinct microbial communities. Nature Communications 11, 1440 (2020). URL http://www.nature.com/articles/s41467-020-15169-0.

[68] Clark, R. L. et al. Design of synthetic human gut microbiome assembly and butyrate production. Nature Communications 12, 3254 (2021). URL http://www . nature.com/articles/s41467-021-22938-y.

[69] Sanchez-Gorostiaga, A., Bajić, D., Osborne, M. L., Poyatos, J. F. \& Sanchez, A. Highorder interactions distort the functional landscape of microbial consortia. PLOS Biology 17, e3000550 (2019). URL https://dx.plos.org/10.1371/journal.pbio. 3000550.

[70] Diagne, N. et al. Ectomycorrhizal diversity enhances growth and nitrogen fixation of Acacia mangium seedlings. Soil Biology and Biochemistry 57, 468-476 (2013). URL https://linkinghub.elsevier.com/retrieve/pii/S0038071712003434.

[71] Laforest-Lapointe, I., Paquette, A., Messier, C. \& Kembel, S. W. Leaf bacterial diversity mediates plant diversity and ecosystem function relationships. Nature 546, 145-147 (2017). URL http://www. nature.com/articles/nature22399.

[72] Shurin, J. B., Mandal, S. \& Abbott, R. L. Trait diversity enhances yield in algal biofuel assemblages. Journal of Applied Ecology 51, 603-611 (2014). URL http://doi.wiley.com/10.1111/1365-2664.12242. 
[73] Georgianna, D. R. \& Mayfield, S. P. Exploiting diversity and synthetic biology for the production of algal biofuels. Nature 488, 329-335 (2012). URL http://www . nature.com/articles/nature11479.

[74] Stadler, P. F. Landscapes and their correlation functions. Journal of Mathematical Chemistry 20, 1-45 (1996). URL http://link.springer.com/10.1007/BF01165154.

[75] Merz, P. Advanced Fitness Landscape Analysis and the Performance of Memetic Algorithms. Evolutionary Computation 12, 303-325 (2004). URL https://direct.mit.edu/evco/article/12/3/303-325/1178.

[76] Szendro, I. G., Schenk, M. F., Franke, J., Krug, J. \& de Visser, J. A. G. M. Quantitative analyses of empirical fitness landscapes. Journal of Statistical Mechanics: Theory and Experiment 2013, P01005 (2013). URL http://stacks.iop.org/1742-5468/2013/i=01/a=P01005?key=crossref . 80c01de42bec35cf69596036ff

[77] Aita, T., Iwakura, M. \& Husimi, Y. A cross-section of the fitness landscape of dihydrofolate reductase. Protein Engineering, Design and Selection 14, 633-638 (2001). URL https://academic.oup.com/peds/article-lookup/doi/10.1093/protein/14.9.633.

[78] Bray, A. J. \& Dean, D. S. The statistics of critical points of Gaussian fields on large-dimensional spaces. Physical Review Letters 98, 150201 (2007). URL http://arxiv.org/abs/cond-mat/0611023. ArXiv: cond-mat/0611023.

[79] Fyodorov, Y. V. \& Williams, I. Replica Symmetry Breaking Condition Exposed by Random Matrix Calculation of Landscape Complexity. arXiv:cond-mat/0702601 (2007). URL http://arxiv.org/abs/cond-mat/0702601. ArXiv: cond-mat/0702601.

[80] Dauphin, Y. et al. Identifying and attacking the saddle point problem in highdimensional non-convex optimization. arXiv:1406.2572 [cs, math, stat] (2014). URL http://arxiv.org/abs/1406.2572. ArXiv: 1406.2572.

[81] Goldford, J. E. et al. Emergent simplicity in microbial community assembly. Science 361, 469474 (2018). URL http://www.sciencemag.org/lookup/doi/10.1126/science.aat1168.

[82] Chang, C.-Y. et al. Engineering complex communities by directed evolution. Nature Ecology \& Evolution 5, 1011-1023 (2021). URL http://www . nature.com/articles/s41559-021-01457-5.

[83] Swenson, W., Wilson, D. S. \& Elias, R. Artificial ecosystem selection. Proceedings of the National Academy of Sciences 97, 9110-9114 (2000). URL http://www.pnas.org/cgi/doi/10.1073/pnas.150237597.

[84] Mueller, U. G. et al. Artificial Microbiome-Selection to Engineer Microbiomes That Confer Salt-Tolerance to Plants. preprint, Plant Biology (2016). URL http://biorxiv.org/lookup/doi/10.1101/081521.

[85] Venturelli, O. S. et al. Deciphering microbial interactions in synthetic human gut microbiome communities. Molecular Systems Biology 14 (2018). URL https://onlinelibrary.wiley.com/doi/abs/10.15252/msb. 20178157. 
[86] Cao, H.-T., Gibson, T. E., Bashan, A. \& Liu, Y.-Y. Inferring human microbial dynamics from temporal metagenomics data: Pitfalls and lessons. BioEssays 39, 1600188 (2017). URL http://doi.wiley.com/10.1002/bies.201600188.

[87] Maynard, D. S., Miller, Z. R. \& Allesina, S. Predicting coexistence in experimental ecological communities. Nature Ecology \&6 Evolution 4, 91-100 (2020). URL http://www.nature.com/articles/s41559-019-1059-z.

[88] Xie, L., Yuan, A. E. \& Shou, W. Simulations reveal challenges to artificial community selection and possible strategies for success. PLOS Biology 17, e3000295 (2019). URL http://dx.plos.org/10.1371/journal.pbio. 3000295.

[89] Shibasaki, S. \& Mitri, S. Controlling evolutionary dynamics to optimize microbial bioremediation. Evolutionary Applications 13, 2460-2471 (2020). URL https://onlinelibrary.wiley.com/doi/10.1111/eva.13050.

[90] Xie, L. \& Shou, W. Steering ecological-evolutionary dynamics to improve artificial selection of microbial communities. Nature Communications 12, 6799 (2021). URL https://www. nature.com/articles/s41467-021-26647-4.

[91] Arias-Sánchez, F. I., Vessman, B. \& Mitri, S. Artificially selecting microbial communities: If we can breed dogs, why not microbiomes? PLOS Biology 17, e3000356 (2019). URL https://dx.plos.org/10.1371/journal.pbio.3000356.

[92] Cui, W., Marsland, R. \& Mehta, P. Effect of Resource Dynamics on Species Packing in Diverse Ecosystems. Physical Review Letters 125, 048101 (2020). URL https://link.aps.org/doi/10.1103/PhysRevLett.125.048101.

[93] Mehta, P., Cui, W., Wang, C.-H. \& Marsland, R. Constrained optimization as ecological dynamics with applications to random quadratic programming in high dimensions. Physical Review E 99 (2019). URL https://link.aps.org/doi/10.1103/PhysRevE.99.052111. 